\title{
Los nuevos instrumentos jurídicos para el desarrollo sostenible del territorio de la Unión Europea
}

\author{
Teresa Parejo Navajas \\ Profesora Ayudante de Derecho Administrativo \\ Universidad Carlos III de Madrid
}

Sumario: 1. EL TERRITORIO COMO ELEMENTO CLAVE PARA EL DESARROLLO SOSTENIBLE DE LAS REGIONES DE EUROPA. 1.1. Principales problemas del territorio como recurso natural. 1.2. Problemas del territorio como soporte de actividades: los desequilibrios regionales. 1.3. Conclusión: necesidad de una Estrategia Territorial a escala europea. 2. LA ORDENACIÓN DEL TERRITORIO EN LA UNIÓN EUROPEA: ACTUALES INSTRUMENTOS Y PERSPECTIVAS DE FUTURO PARA EL DESARROLLO DEL TERRITORIO EN EUROPA. 2.1. Planificación territorial europea formal: las políticas sectoriales territoriales. 2.2. Planificación territorial europea material: la Estrategia Territorial Europea como instrumento soft de ordenación del territorio. 2.3. El futuro de la planificación europea del territorio: opciones para una ordenación del territorio a escala europea a la luz del Derecho originario actual y en ciernes. a) Opciones A: ordenación del territorio formal a través de la cohesión territorial. b) Opción B: ordenación del territorio material a través de instrumentos soft y del MCA. 3. CONCLUSIÓN: EL MODELO DE ORDENACIÓN DEL TERRITORIO A PARTIR DE UNA OPCIÓN DE NATURALEZA ECLÉCTICA.

La importancia de la ordenación del territorio como técnica para lograr el desarrollo territorial sostenible por un lado, y las dificultades reales para desarrollar un plan a gran escala por ese carácter estratégico del territorio en cada Estado miembro, por otro, han provocado un desajuste entre la magnitud real de tal proyecto y las técnicas otorgadas a las instituciones comunitarias para desarrollarlo. Efectivamente, el derecho originario de la Unión Europea (UE) no contempla una competencia formal en la materia que justifique el desarrollo de una política a escala europea. Sin embargo, la UE transforma el territorio a través de las políticas sectoriales para las que el Tratado sí le reconoce competencia. Es precisamente la falta de fundamento jurídico formal que ampare una competencia en materia de ordenación del territorio comunitaria lo que ha provocado que se desarrollen nuevos instrumentos jurídicos, al margen de los tradicionales, que den respuesta a esa realidad transformadora. 


\section{EL TERRITORIO COMO ELEMENTO CLAVE PARA EL DESARROLLO SOSTENIBLE DE LAS REGIONES DE EUROPA}

El territorio de la UE no es un elemento inmutable; ha experimentado una enorme transformación desde mediados del siglo Xx por diferentes razones, condicionando sus características naturales, y que pueden resumirse de la siguiente manera: a) cambios políticos ocurridos en los Estados miembros tras la Segunda Guerra Mundial, y con ellos, cambios en las definiciones de sus fronteras, y por tanto, de sus territorios; b) cambios geográficos del territorio de la UE (ampliación del mismo) debido a las sucesivas adhesiones; c) cambios, asimismo, en la configuración institucional y competencial de la UE debido a esos cambios geográficos; d) cambios en la población como consecuencia de la nueva situación política de la UE; y e) la creciente importancia del territorio como base fundamental para el desarrollo de las políticas sectoriales. Estos cambios en el territorio afectan, también, a los elementos de desarrollo económico asociados al mismo en tanto que soporte de las actividades antrópicas, dificultando el logro de los objetivos establecidos en el art. 2 del Tratado de la Comunidad Europea (TCE), en particular, los que se refieren al «(...) desarrollo armonioso, equilibrado y sostenible de las actividades económicas en el conjunto de la Comunidad, (...) un alto nivel de protección y de mejora de la calidad del medio ambiente, la elevación del nivel y de la calidad de vida, la cohesión económica y social y la solidaridad entre los Estados miembros», esto es, en definitiva, el desarrollo territorial sostenible de las regiones de Europa.

La corrección de las disparidades territoriales de las regiones de la UE no sólo es una prioridad política de la UE, sino que forma parte, tras su inclusión en el Tratado, del acervo comunitario, que constituye la base de derechos y obligaciones de los Estados miembros y que, por tanto, debe ser respetado y desarrollado por todos ellos.

\subsection{Principales problemas del territorio como recurso natural}

La ampliación europea y, en general, los cambios geográficos producidos en el territorio de la UE a lo largo de su historia, no suponen las únicas dificultades para el logro de los objetivos del art. 2 TCE asociados al territorio en tanto que elemento natural. Otros problemas, por su importancia, han propiciado también la actuación de la Comunidad:

a) Desertificación. Degradación y/o destrucción del suelo:

El territorio, tal y como señala la Carta Europea del Suelo de 1972, es uno de los bienes más preciosos de la humanidad en tanto que soporte de todas sus actividades, así como el medio natural que permite la vida. Sin embargo, se 
LOS NUEVOS INSTRUMENTOS JURÍDICOS PARA EL DESARROLLO SOSTENIBLE...

trata de un bien escaso y fácilmente destruible y por ello, su ordenación se convierte en una actividad de carácter estratégico de suma importancia para el desarrollo de las regiones de Europa e, incluso, para la propia existencia de la humanidad: aporta el agua y los nutrientes necesarios para el desarrollo de la agricultura, juega un papel principal en la protección y conservación natural del agua y en el intercambio de gases en la atmósfera, etc y, además, es el soporte del patrimonio natural y cultural de los pueblos de Europa.

Todas estas características explican la importancia de la protección del suelo y de la necesidad de que tan protección se ejerza tanto dentro de la UE como fuera de sus fronteras. Así, a escala internacional, la Declaración de Río de 1992 logró que los Estados participantes adoptaran una serie iniciativas que evidenciaban la importancia de la protección del suelo para prevenir y reducir su degradación, para rehabilitar los suelos degradados. En el ámbito de la UE, el Sexto Programa de Acción Medioambiental establece (para un período de vigencia de diez años y desde el año 2001) un objetivo de protección y restauración del funcionamiento de los sistemas naturales para detener la pérdida de biodiversidad en la UE y en el mundo; proteger los suelos contra la erosión y la contaminación, como parte de una Estrategia de Desarrollo Sostenible ${ }^{1}$.

La degradación del suelo ${ }^{2}$, esto es, la transformación del suelo productivo en no productivo (desierto) puede estar provocada tanto por procesos naturales (desertización) como por la acción del hombre (desertificación), principalmente por: 1) sobre explotación del suelo; 2) destrucción de la escasa vegetación existente en zonas áridas; 3) incendios intencionados; 4) salinización debido a prácticas de riego incorrectas; y 5) aumento de la población $\mathrm{y}$, por ende, de la actividad antrópica sobre el mismo ${ }^{3}$.

Aunque la degradación del suelo es un problema global, en Europa, por su clima y grado de desarrollo, tiene especial relevancia. Los métodos de la agricultura tradicional, la erosión y los procesos de compactación de los suelos, tienen importantes repercusiones sobre la calidad del territorio de los Estados miembros, lo que provoca transformaciones en los paisajes y en los modelos de desarrollo económico de las regiones, especialmente las rurales, produciendo su degradación económica y social ${ }^{4}$.

\footnotetext{
${ }^{1}$ Comunicación de la Comisión al Consejo, al Parlamento Europeo, al Comité Económico y Social y al Comité de las Regiones sobre el Sexto Programa de Acción de la Comunidad Europea en materia de Medio Ambiente «Medio ambiente 2010: el futuro está en nuestras manos», de 24 de enero de 2001. COM (2001) 31 final http://eur-lex.europa.eu/LexUriServ/site/es/com/2001/com2001_ 0031es01.pdf (may 2005). p. 4.

${ }^{2}$ Report SCAPE (Soil Conservation and Protection for Europe) desertification workshop. Schruns (AT) 12/10/2004. http://www.scape.org/Schruns_desertification.pdf (may 2005). p.1.

${ }^{3}$ Para mayor información, vid. http://www.botany.uwc.ac.za/Envfacts/facts/desertification.htm (mayo 2005).

${ }^{4}$ Most EU countries are affected by this problem to some extent. In the Mediterranean area, soil erosion is very severe, moderately to seriously affecting $50 \%$ to $70 \%$ of agricultural land. Conventional
} 


\section{b) Despoblación:}

La despoblación supone la disminución dramática de los habitantes de una región. Sin embargo, también la huída de una parte (o sector determinado) de esa población (p.e. la población más joven) puede provocar, a veces, los mismos efectos que una despoblación masiva o iniciar el proceso de despoblación total de una región. Aunque generalmente la despoblación se produce en regiones con importantes desventajas geográficas, el problema también puede producirse en otras, al menos de una manera indirecta. En cualquier caso, todas las regiones de la UE tienen alguno de los siguientes problemas asociados a la despoblación: aislamiento geográfico, disminución demográfica, escasa actividad económica, disminución de los niveles de renta y, con ello, de las condiciones de vida de sus habitantes y de los niveles de empleo. En muchas ocasiones, estos problemas se dan al mismo tiempo, provocando el éxodo rural hacia las zonas urbanas y, una vez más, la disminución del desarrollo económico de las regiones.

Por lo tanto, y tal y como confirma la Dirección General de Política Regional de la Comisión, la despoblación es un problema en alza ${ }^{5}$ : la gran mayoría de las regiones de la UE, bien perderán población en favor de aquellas otras que la reciban, bien sufrirán un proceso de transformación interno producido por la concentración de la población en unas determinadas zonas (urbanas) en detrimento de otras (rurales). Esta situación, si continúa en esta línea, como es previsible de otro lado, agudizará las disparidades ya existentes entre las diferentes regiones dificultando más aún la consecución de los objetivos del Tratado.

\section{c) Escasez del suelo por sobreexplotación}

La desertización y la desertificación de los suelos, debido tanto a la acción de la naturaleza como a la acción del hombre por sobreexplotación, respectivamente (o a ambas al tiempo, que es lo que normalmente sucede), provocan el empobrecimiento de este recurso natural y como consecuencia de ello, su destrucción irreparable, pues la naturaleza no es capaz de lograr su regeneración paulatina al ser más rápido y agresivo el proceso de su destrucción.

Los problemas provocados por el empobrecimiento del suelo no sólo tienen repercusiones sobre la propia naturaleza (pérdida de biodiversidad, desertificación, erosión, destrucción de los paisajes, etc), sino también sobre la economía de los países afectados. La falta de riqueza de los suelos provoca

\footnotetext{
agriculture intensification (increased mechanisation and ploughing) over the past 50 years has contributed substantially to this trend, increasing the risk of desertification in the most vulnerable areas. http:/ /www.ecaf.org/English/First.html\#3.a. The erosion increases agricultural production costs by about $25 \%$ each year (53 EUR per hectare). Further, if on-site and off-site costs are combined, the total annual cost of erosion from agriculture can be estimated at about 85.5 EUR per hectare. http://www.ecaf.org/English/First.html\#3.a.
}

${ }^{5}$ http://ec.europa.eu/comm/regional_policy/newsroom/document/pdf/newsletter/88_01_es.pdf 
LOS NUEVOS INSTRUMENTOS JURÍDICOS PARA EL DESARROLLO SOSTENIBLE...

la aparición de un efecto imparable y sucesivo de empobrecimiento (empobrecimiento del suelo y de los recursos naturales en general, que da lugar al empobrecimiento económico, que da lugar al empobrecimiento demográfico por inmigración, que da lugar a más empobrecimiento económico, y así sucesivamente) y que finalmente lleva a la aparición o a la agudización de los problemas relacionados con los desequilibrios regionales.

Estos terribles efectos que la actividad del hombre tiene sobre el territorio son, cabalmente, perversos pues en gran medida están provocados por las mismas actividades que crean riqueza y, por tanto, disminuyen las diferencias entre las regiones. Y estas diferencias regionales existen en Europa desde que se produjeron las primeras adhesiones a las entonces denominadas Comunidades Europeas y persisten hasta hoy a pesar de los esfuerzos de la política de cohesión económica y social desarrollada a través del Fondo de Desarrollo Regional (FEDER) desde su creación en 1975.

\subsection{Problemas del territorio como soporte de actividades: los desequilibrios territoriales}

El territorio es, también, el soporte de las actividades del hombre. Estas actividades tienen un doble efecto de transformación sobre el mismo: por un lado, en términos materiales, transformando paisajes, creando ciudades, etc. $y$, por otro, aunque también al mismo tiempo, en términos económicos, provocando el desarrollo económico de las regiones.

De esta manera, la transformación física o material del territorio a través de inversiones económicas enriquece las regiones en las que se producen. La diversidad en el desarrollo regional en términos económicos se traduce en diferencias importantes en lo que al nivel y calidad de vida se refiere de sus habitantes, cuya superación progresiva es posible, desde la creación de la política de Cohesión comunitaria en el Acta Única Europea en 1986 y su consolidación como objetivo esencial de la UE en el Tratado de la Unión Europea en 1992, que actualmente se encuentra recogido en el art. 2 TCE.

El medio más importante para la consecución de la Cohesión entre las regiones europeas es el de la intervención financiera estructural a través de los Fondos Estructurales y de otros instrumentos financieros comunitarios, entre los que está el Banco Europeo de Inversiones. Las intervenciones específicas de los Fondos Estructurales se materializan en el territorio de los estados miembros a través de la programación correspondiente, según se trate de una zona geográfica comprendida en el objetivo 1, en el objetivo 2 o en el objetivo 3, así como a través de iniciativas comunitarias definidas por los artículos 20 y 21 del Reglamento 1260/1999, de 21 de junio de 1999. Una vez determinadas las zonas subvencionables, se establecen los programas 
comunitarios a partir de iniciativas nacionales y comunitarias, y de acciones innovadoras, con el fin de lograr los objetivos generales y prioritarios del Reglamento 1260/1999, y el del art. 2 TCE de desarrollo armonioso, equilibrado y sostenible de las regiones de Europa.

\subsection{Conclusión: necesidad de una Estrategia a escala europea}

La unidad del territorio como escenario de la intervención del ser humano y su escasez como recurso, apunta a una lógica y necesaria consideración global del impacto de las intervenciones del hombre en dicho recurso. En efecto, toda intervención humana en el territorio supone una «organización» del mismo.

El uso de un bien escaso sólo puede admitirse si el grado de disponibilidad del recurso es tal que no condiciona desarrollo futuros de otras actividades u oportunidades (en términos de sostenibilidad). Pero esto no es posible en el territorio. Por tanto, la consideración global de la intervención sobre el territorio (a escala europea) supone un verdadero presupuesto lógico de su uso y distribución eficiente o justa.

Así, los problemas del territorio pueden y deben resolverse a través de acciones concertadas en el nivel comunitario. Es cierto que la UE no tiene competencia en materia de ordenación del territorio y que, por tanto, son los Estados miembros los competentes, al menos hoy por hoy, para elaborar sus propias estrategias territoriales dando solución a todos esos problemas que afectan a su específico territorio, si bien condicionados por la normativa europea sectorial de obligado cumplimiento en virtud del principio de primacía del derecho comunitario. Sin embargo, la propuesta a nivel europeo de políticas y medidas (art. $3 \mathrm{TCE}$ ), intercambio de experiencias, intercambio de buenas prácticas y desarrollo de una red de acciones innovadoras son de importancia primordial no sólo a los efectos de la formulación de una política nacional de ordenación del territorio en los Estados miembros, sino también para la selección de las prioridades comunitarias en relación con el objetivo de Cohesión y de su implementación a través de los proyectos financiados por el FEDER.

Existen ejemplos de acciones comunes a través de estrategias a escala europea: en relación con la cohesión económica y social y el desarrollo sostenible en términos puramente medioambientales -ámbitos en los que el Tratado sí le otorga a la UE competencias- esas acciones comunes se han traducido en estrategias europeas en favor del desarrollo sostenible. Así, por ejemplo, la propuesta de la Comisión al Consejo Europeo celebrado en Gotemburgo en junio de 2001, sobre una Estrategia Europea de Desarrollo Sostenible en la que se propone la coordinación de las políticas sectoriales para lograr el desarrollo sostenible a partir del equilibrio económico, social 
y medioambiental de las medidas adoptadas ${ }^{6}$. Esta propuesta ha sido incluida en las perspectivas financieras de la comunidad, según se desprende de la Comunicación al Consejo y al Parlamento Europeo «Construir nuestro futuro común - Retos políticos y medios presupuestarios de la Unión ampliada (2007-2013)» ${ }^{7}$, complementada por la Comunicación de la Comisión al Parlamento Europeo «Perspectivas financieras 2007-2013 $»^{8}$. Por tanto, tanto la política de medio ambiente (perspectiva medioambiental del desarrollo sostenible) como la política de cohesión para el crecimiento y el empleo (perspectiva social del desarrollo sostenible), quedarán integradas en las diferentes políticas prioritarias y se financiarán a través de los fondos europeos ${ }^{9}$.

Pero la política territorial es, entre todas las políticas públicas, la que más se preocupa de aportar algo a los objetivos comunitarios de cohesión económica, social y territorial, de desarrollo sostenible, y de mejora de la competitividad entre los Estados miembros (HILDEBRAND SCHEID, 1999) ${ }^{10}$, afirmación que también se extrae de la definición que de la ordenación del territorio realiza la Carta Europea de Ordenación del Territorio de 1983. Estos objetivos, en la década de los años noventa, se erigieron con fuerza como referentes del desarrollo regional ${ }^{11}$, por lo que, aquellas regiones que llevaran y lleven

\footnotetext{
${ }^{6}$ Para mayor información sobre el Consejo Europeo de Gotemburgo, véase http:// eur-lex.europa.eu/ LexUriServ/ site/ es/ com/2001/ com2001_0264es01. pdf (mayo 2006)

7 COM (2004)101, de 10 de febrero de 2004

${ }^{8}$ COM (2004)487, de 14 de julio de 2004

9 Para mayor detalle, http://www.mma.es/polit_amb/fondos/redauto/pdf/cohesion2007_2013.pdf

${ }^{10}$ Es la política territorial, término prácticamente sinónimo de ordenación del territorio, la que más se preocupa de aportar algo a los tres objetivos citados: 1) a la cohesión económica, social y territorial contribuye a través del sistema de jerarquía de ciudades que organiza el acceso de los ciudadanos a los equipamientos básicos, infraestructuras urbanas y servicios públicos y privados en condiciones equivalentes; 2) al desarrollo sostenible a través de la coordinación y compatibilización entre sí de los diferentes usos del suelo; y 3) a la mejora de la competitividad, mediante su impulso a la formación de redes de cooperación entre ciudades. Hildebrand Scheid, A. Política territorial y desarrollo regional en España y Europa: Una visión comparad en vísperas del siglo XXI. Desarrollo Local y desarrollo regional frente al siglo XXI. Ciudad y Territorio. Estudios Territoriales. Vol. XXXI. Tercera Época. No 122, invierno 1999. pp. 785-807.
}

11 Parejo Alfonso propone una definición de política regional apuntando previamente, no obstante, que dicha expresión dista mucho, al menos en el plano internacional y, desde luego, en el europeo, de tener una significación clara y pacífica por la variedad que presentan las políticas estatales o nacionales, tanto en objetivos y contenidos, como en instrumentos y procedimientos. Así, con esta precisión, entiende que se trata de la política que «para ser tal no le basta con tener un (incluso importante) impacto territorial acotado (léase regional), siéndole preciso tener directamente por objeto propio la resolución de los problemas estructurales de un área determinada o, con carácter general, la superación progresiva de las diferencias de desarrollo existentes entre unos y otros territorios (regiones)». PAREJO Alfonso, L. La política regional en la Comunidad Económica Europea.; incluido en GARCía DE ENTERRÍA, E.; González Campos, J. D., y Muñoz Machado, S. «Tratado de Derecho Comunitario Europeo». Volumen III. Cívitas, $1^{\circ}$ edición. Madrid, 1986, pp. 573 y ss. KRÄMER la define como aquélla que pretende fomentar el desarrollo armonioso de las regiones a través de la financiación que le proporcionan los Fondos Estructurales, que fueron creados por el Consejo mediante el Reglamento 2052/ 88, sobre los Fondos Estructurales, sustituido posteriormente por el Reglamento 2081/93, ambos sobre la base jurídica del antiguo $130 \mathrm{D}$ (actual art. 160) del Tratado de la Comunidad. KRÄMER, L. «Derecho Ambiental y Tratado de la Comunidad Europea». Marcial Pons. Barcelona, 1999, p. 55. 
a cabo un modelo de desarrollo económico, social y ecológico-territorial que corresponda a una razonable combinación de estos tres objetivos, que son, además, interdependiente, serán y son las que, probablemente, a largo plazo, sean verdaderamente competitivas ${ }^{12}$.

\section{Por lo tanto, la ordenación del territorio es un instrumento estratégico} - en su doble dimensión de política y de competencia- de enorme relevancia, pues a través del mismo se condiciona el desarrollo de una zona a medio y largo plazo, tanto en términos territoriales - cambios en los usos del suelo y del paisaje de los Estados miembros- como económicos - modificaciones en la competitividad de las regiones en las que dichas políticas son desarrolladas, así como su situación económica relativa dentro del espacio global europeo-13.

\footnotetext{
12 Además de las anteriores precisiones sobre el concepto de política regional, quizá resulte interesante destacar, además, la diferencia entre desarrollo regional y desarrollo local propuesta por LázARo ARAujo. Según éste, «resulta imposible encontrar aportaciones teóricas que intenten, y menos aún que consigan, trazar la linde que separa conceptualmente el desarrollo local del regional (...). Aunque trabajos hay cuyos títulos parecen sugerir una aclaración de tales diferencias, al poco de sumergirse en su lectora, puede uno percatarse de que, si ese era el propósito, no se ha conseguido». (...) En todo caso, «ante el reiterado e indistinto recurso a expresiones como «desarrollo regional y local» o «desarrollo regional o local», se hace muy difícil sustraerse a la tentación de negar que se trate de conceptos diferentes. (...) Los especialistas en economía regional más reputados reconocen sin complejos que no hay teorías específicas para el desarrollo regional, sino adaptaciones de las concepciones sobre desarrollo nacional (...) Tal vez suceda que, en materia de desarrollo, la única diferencia sustancial sea la escala territorial de las actuaciones (...). Probablemente ahí estribe la diferencia entre desarrollo regional y desarrollo local, en las estrategias e instrumentos a aplicar en cada caso (...). Hay diferentes niveles territoriales del desarrollo (supranacional, nacional, regional y local) que no se contraponen, antes bien, se complementan (...). La política regional a escala nacional perseguirá el reequilibrio y la solidaridad interregional. La política regional autonómica, llevada a cabo por los gobiernos regionales, tendrá sus efectos a escala interregional. A su vez, se verá apoyada, complementada y cimentada en el desarrollo local. El mismo camino, con consideraciones inversas, puede recorrerse desde abajo hacia arriba. (...) Este es un tema muy apropiado para invocar el principio de subsidiariedad (...)», que deberá aplicarse sin renunciar a la «práctica de fórmulas de cooperación interadministrativas». LÁZARO ArAUjo, L., El desarrollo local y los Fondos Estructurales. Ciudad y Territorio. Estudios Territoriales, Vol. XXXI, Tercera época, $\mathrm{n}^{\circ}$ 122, 1999. Pag. 735 a 755.
}

${ }^{13}$ En este sentido, la creación del Parque Natural del Delta del Ebro, se inicia a principios de los años ochenta, como reacción a un conjunto de actuaciones locales de desarrollo agrícola y turístico, provocando un conflicto entre intereses puramente económicos y los medioambientales. El establecimiento del Parque Natural ha evitado la ejecución de la mayoría de las actuaciones que representaban una amenaza para los espacios protegidos $\mathrm{y}$, a la vez, ha permitido el desarrollo de determinadas actividades tradicionales. Todo ello, ha conducido hacia una mejora significativa de la calidad ambiental y de las funciones ecológicas del Delta. Manuel, D., y Martí, N. El Parque Natural del Delta del Ebro. Incluido en Aguilar, S., Font, N. y Subirats, J. «Política ambiental en España. Subsidiariedad y desarrollo sostenible. Tirant lo Blanch. Valencia, 1999, pp. 145 y ss. Por otro lado, en el caso de los Túneles de Vallvidriera, se tomó la decisión de construir una importante infraestructura viaria en respuesta a los intereses socioeconómicos — proyección de Barcelona hacia el Vallés Occidental y hacia Francia - y políticos — legitimación de la Generalitat — a la que se oponen actores de ámbito local con argumentos medioambientales, pues los túneles discurrirían a través de una montaña con un rico biotipo de fauna y flora mediterránea. FrancESCA GoMILA, M., Algunas reflexiones en torno a cuatro experiencias de protección ambiental. Incluido en Aguilar, S.; Font, N. y SubiRAts, J., «Política ambiental en España. Subsidiariedad y desarrollo sostenible. Tirant lo Blanch. Valencia, 1999, pp. 25 y ss. En ambos casos, las decisiones en materia de política medioambiental y sobre infraestructuras de transportes, respectivamente, suponen decisiones de política territorial en tanto que estructurantes de los territorios sobre los que actúan. En Parejo NAvajas, T., «La Estrategia Territorial Europea: la percepción comunitaria del uso del territorio».Marcial Pons, 2004. 
LOS NUEVOS INSTRUMENTOS JURÍDICOS PARA EL DESARROLLO SOSTENIBLE...

Sin embargo, cuando las acciones son propiamente de planificación territorial, como la UE no tiene competencia, no ha podido desarrollar Estrategias a escala europea teniendo que buscar mecanismos alternativos para justificar jurídicamente su acción. De esta manera, conviven al mismo tiempo la ordenación del territorio comunitaria sectorial o desarrollada a través de las políticas sectoriales territoriales, con otras técnicas de naturaleza peculiar surgidas al amparo precisamente de su falta de encaje jurídico competencial formal, y que se refieren a Estrategias que se sustentan sobre la base de documentos de carácter soft, y que se analizan más adelante. Esta compleja situación compuesta por acciones e iniciativas que afectan al territorio tomadas en los diferentes niveles territoriales y, como consecuencia de ello, por instrumentos de diversa naturaleza jurídica, hace necesaria una visión global a escala europea que coordine todas estas acciones y controle la actividad generada por todos los entes territoriales. Esa visión global es la que proporciona la elaboración de una Estrategia territorial (y también, como no, otras sectoriales, que ahora no interesan) a escala europea.

\section{LA ORDENACIÓN DEL TERRITORIO EN LA UNIÓN EU- ROPEA: ACTUALES INSTRUMENTOS Y PERSPECTIVAS DE FUTURO PARA EL DESARROLLO DEL TERRITORIO EN EUROPA}

La inquietud sobre la organización del territorio está presente con especial eficacia e interés en la UE desde los años 80, con la firma de la Carta Europea de Ordenación del Territorio. En ella, por primera vez, los Estados firmantes europeos se ponen de acuerdo sobre el concepto de ordenación del territorio válido para todos ellos. Este primer paso resultó de enorme importancia, pues hasta ese momento la puesta en marcha de una Estrategia territorial a escala europea resultaba imposible de llevar a cabo, principalmente porque cada país manejaba un concepto diferente de ordenación del territorio. A partir de este hito, las iniciativas relacionadas con la planificación del territorio en Europa se fueron sucediendo con cierta rapidez en el tiempo, pudiéndose diferenciar dos bloques en función de su encaje jurídico dentro del Tratado. Así están 1) las iniciativas comunitarias amparadas en el desarrollo de las diferentes políticas sectoriales del art. 3 TCE (con encaje formal competencial, por tanto); y 2) las iniciativas, más novedosas, amparadas en lo que se denomina, en derecho anglosajón, el soft law (iniciativas no imperativas por no tener, en este caso, encaje competencial formal, pero de indudable eficacia práctica, tal y como se verá más adelante). 


\subsection{Planificación Territorial europea formal: las políticas secto- riales territoriales}

Las políticas sectoriales del art. 3 TCE, competencia de la Comunidad, dirigidas a la consecución de los objetivos de cohesión económica y social y de desarrollo, en definitiva, sustentable del territorio, del art. 2 TCE, cercenan, muchas veces, el recurso natural que sirve de apoyo a todas esas actividades de potenciación económica. Otras veces, sin embargo, contrarrestan estos efectos destructivos. En este sentido, entre las políticas sectoriales que producen efectos sobre el territorio se pueden distinguir: 1) las políticas desarrolladas para mitigar los efectos perniciosos de la acción del hombre sobre el territorio (política de medioambiente o la de cohesión); y 2) las políticas cuyo fin último es el desarrollo económico de las regiones comunitarias (redes transeuropeas, política agrícola común, política de turismo, etc.).

Por ello, una de las formas de transformación «planeada» u «ordenada» del territorio por la UE es la que le permite el propio desarrollo de las políticas sectoriales, que, de esta manera, pueden denominarse, «políticas sectoriales territoriales», ya que con su puesta en marcha, determinan el modelo de desarrollo territorial de la UE. Todas ellas, en cualquier caso, buscan el incremento de la calidad de vida y, desde la creación del FEDER, y con éste, la política regional comunitaria, la consecución de la igualdad de oportunidades económicas y sociales de todas las regiones de Europa (objetivos del art. 2 TCE).

\subsection{La Planificación Territorial europea material: la Estrategia Territorial Europea como instrumento soft de ordenación del territorio}

Al mismo tiempo que iba incrementando sus competencias en el proceso de construcción europeo y, seguramente, con el ánimo de coordinar todas esas acciones sectoriales independientes, la UE fue buscando nuevas vías para llevar a cabo más eficazmente el objetivo de desarrollo territorial sostenible del art. 2 TCE, que es, en definitiva, la organización coherente de las políticas sectoriales del art. 3 TCE.

Sin embargo, al no contemplarse en el Tratado una competencia expresa en materia de ordenación del territorio, ni tampoco poder justificar, al menos hoy por hoy, el desarrollo de una tal política al amparo de la regulación existente, se fueron desarrollando una serie de documentos (de carácter general: Europa 2000, Europa 2000 +; y de carácter específico de determinadas zonas sensibles: programas Terra $^{14}$ y Gestión Integrada de las Zonas Costeras

${ }^{14}$ Véase el programa Terra en http://www.interreg-mac.org/es/doc/normativa/terra.pdf 
LOS NUEVOS INSTRUMENTOS JURÍDICOS PARA EL DESARROLLO SOSTENIBLE...

$\left.(\mathrm{GIZC})^{15,16}\right)$-, así como la más reciente Estrategia Temática para el Medio Ambiente Urbano ${ }^{17}$, entre otros, cuyo objetivo principal se refiere al desarrollo sostenible del territorio de la UE. De las conclusiones desarrolladas a partir de la puesta en marcha de estos programas, surgieron otros que, a diferencia de los anteriores, afrontan ya directamente la planificación del territorio a escala europea, aunque a través de técnicas de naturaleza jurídica soft, en el cumplimiento del principio de atribución de competencias (en este caso, por la falta de competencia en materia de ordenación del territorio).

El término soft law fue acuñado por Lord McNair para el ámbito del derecho internacional (RIEDEL, 1991) ${ }^{18}$ y es en este ámbito en el que mayor desarrollo ha experimentado. No obstante su relevancia, no existe una clara y única definición del término. Por ello, resulta necesario señalar en este punto que la que aquí va a manejarse es la utilizada en el derecho comunitario. En este sentido, tal y como señala Linda SENDEN, el soft law se refiere a las «reglas de conducta establecidas en una serie de instrumentos a los que no se le han atribuido fuerza vinculante pero que tienen un cierto efecto jurídico (indirecto) y que producen, en todo caso, efectos prácticos». Es por ello por lo que se define, asimismo, como aquél derecho que no puede enmarcarse dentro del denominado hard law (SENDEN, p. 112) ${ }^{19}$.

A partir de este concepto se pueden distinguir, siguiendo al profesor AlONSO GARCíA, entre soft law ad intra y ad extra (Alonso GARCÍA, pp. 71-80). El primero de ellos es el que se refiere a aquellos mecanismos establecidos por el derecho comunitario para concretar las relaciones entre las instituciones en el seno de la Comunidad. El segundo de ellos, es el que se refiere al principio de confianza mutua o cooperación leal de los Estados miembros con la Comunidad y que se encuentra expresamente recogido en el art. 10 TCE. Los instrumentos que más adelante se analizarán se ajustan a este segundo tipo de soft law, puesto que precisamente surgieron como consecuencia de la cooperación entre los Estados miembros y la Comunidad.

Según se establece en el art. 249.1 TCE, para el cumplimiento de su misión, las instituciones de la Comunidad Europea cuentan con instrumentos de

\footnotetext{
15 Véase el documento de reflexión sobre el programa GIZC en http://ec.europa.eu/ environment/iczm/ pdf/ exsum_es. pdf

16 Para un análisis más detallado de los documentos, véase PAREjo NAVAJAS, T., «La Estrategia Territorial Europea: la percepción comunitaria del uso del territorio». Marcial Pons, Madrid, 2004.

17 COM (2005) 718 final, de 11.1.2006.

18 Vid. Introduction Prefacio de Lord McNair de la obra D.P. O’Conell. Internacional Law. Vol. I (1970) 3 et. Seq. citado por Riedel, E., Standards and sources. Farewell to exclusivity of the Sources triadin International Law. EJIL (1991) 58. http://www.ejil.org/journal/Vol2/No2/art3.pdf.

${ }^{19}$ En lo que a la relación del soft law con el hard law se refiere, véase el artículo del profesor (ALONSO GARCÍA, R, 2001; pp. 71-80), especialmente el punto que se refiere al soft law como alternativa al hard law, esto es, cuando no se dan las circunstancias necesarias para ulteriores intervenciones de naturaleza imperativa, bien por motivos coyunturales, bien por no existir competencia en la materia, como es el caso que nos ocupa.
} 
intervención vinculantes y no vinculantes, lo que no impide, sin embargo, su calificación como instrumentos jurídicos integrantes del ordenamiento comunitario. Por tanto, y tal y como señaló el Tribunal de Justicia en su sentencia de 13 de diciembre de 1989, asunto $\mathrm{n}^{\mathrm{o}} 322 / 88^{20}$, la ausencia de efectos vinculantes no implica per se ausencia de efectos jurídicos (AlONSO GARCÍA, p. 64).

El artículo 249 TCE, sin embargo, no contempla todos los actos que pueden ser adoptados por las instituciones comunitarias. Así, existen otros, que por no encontrarse entre los «enumerados», son calificados por la Doctrina como actos atípicos, y como tales, no tienen ni la naturaleza ni los efectos de los actos del art. 249 TCCE, ni están sometidos al mismo régimen de adopción $(\mathrm{CONV} 449 / 02, \text { p.2) })^{21}$.

Los actos atípicos o innominados, son aquellos actos de las instituciones u órganos comunitarios, de naturaleza diversa, que no están sistematizados en los Tratados en cuanto a su denominación, alcance o estructura. Estos actos emanan del Consejo, de la Comisión o de varias instituciones comunitarias al mismo tiempo, y según el Tribunal de Justicia, tienen legitimidad y carácter obligatorio $^{22}$, en algunos casos. Por tanto, este tipo de actos no son necesariamente obligatorios ni vinculantes. Sin embargo, en la práctica, la falta de imperatividad formal no ha impedido que los actos atípicos, como los que a continuación se van a explicar, produzcan efectos jurídicos.

\section{a) La Estrategia Territorial Europea (ETE): descripción del documento}

La ETE representa, a fecha de hoy, la culminación de un proceso que ha dado lugar a la formalización de la perspectiva europea del territorio. Supo-

\footnotetext{
20 «Las recomendaciones, que, a tenor del párrafo 5 del artículo 189 del Tratado, no serán vinculantes, son generalmente adoptadas por las instituciones comunitarias cuando éstas no ostentan, con arreglo al Tratado, la potestad de dictar actos obligatorios, o cuando consideran que no procede dictar normas de mayor fuerza vinculante. Dado que constituyen actos que no pretenden producir efectos obligatorios, ni siquiera para sus destinatarios, no pueden, por sí mismos, crear derechos para los justiciables que éstos puedan ejercitar ante los órganos jurisdiccionales nacionales. Sin embargo, dado que las recomendaciones no pueden considerarse como actos carentes de todo efecto jurídico, los órganos jurisdiccionales nacionales deben tenerlas en cuenta a la hora de resolver los litigios de que conocen, sobre todo cuando aclaran la interpretación de aquellas disposiciones nacionales que fueron adoptadas con la finalidad de garantizar su aplicación, o también cuando tienen como finalidad completar las disposiciones comunitarias que tienen carácter obligatorio». Sentencia del Tribunal de Justicia (Sala Segunda), de 13 de diciembre de 1989, asunto no 322/88, Salvatore Grimaldi contra Fonds des Maladies Professionnelles, p. 1. http://curia.eu.int/en/recdoc/indexaz/index.htm.

${ }^{21}$ Según ha señalado la Convención Europea en relación con la simplificación de los procedimientos e instrumentos «Se mantienen los actos denominados «atípicos» (conclusiones, resoluciones, comunicaciones...) para responder a una necesidad de flexibilidad, pero el recurso a los mismos debería limitarse a los casos en que no esté previsto recurrir a un acto formal».
}

${ }^{22}$ STJCE de 3-2-1976, Manghera, as. 59/75, Rec. 91 y 8-4-1976, Defrenne, as. 43/75, Rec. 455. http:/ /curia.eu.int/en/recdoc/indexaz/index.htm. 
LOS NUEVOS INSTRUMENTOS JURÍDICOS PARA EL DESARROLLO SOSTENIBLE...

ne, así, una etapa en un proceso de progresiva maduración a partir del impacto territorial que provocan las decisiones comunitarias sobre política sectorial y del condicionamiento que ese mismo impacto territorial tiene para la satisfacción de los objetivos comunitarios de desarrollo equilibrado.

Con la aparición de la visión horizontal de los problemas en la Comunidad Europea que tuvo su primera evidencia en el desarrollo de la política regional comunitaria - luego de cohesión social y económica- y con el consecuente aumento de la cooperación transfronteriza, se inició un nuevo espacio de reflexión entre las instituciones comunitarias, así como en el seno de algunas organizaciones internacionales — como el Consejo de Europaque fructificaron en la elaboración de una serie de documentos e informes, como ya se ha indicado, que evidenciaron ese interés por organizar el territorio conforme a unos criterios comunes a todos los Estados miembros para lograr la corrección de los principales desequilibrios de sus regiones, entre los que destaca de manera clara, el de la ETE.

La versión última del documento sobre la ETE fue acordada en la reunión informal de Ministros responsables en materia de ordenación del territorio en Postdam, en mayo de 1999 y se estructura en dos partes (A y B): la Parte A, titulada «Para un desarrollo equilibrado y sostenible del territorio de la Unión Europea: la contribución de la política de desarrollo territorial», establece los aspectos de carácter político del documento, las orientaciones, recomendaciones y sus posibles formas de aplicación; y la Parte B, titulada «El territorio de la Unión Europea: tendencias, perspectivas y retos», en la que se hace una prospectiva de las líneas de desarrollo territorial de la Unión Europea mediante la relación de datos y características analíticas del territorio europeo.

No obstante la estructura del documento, su contenido puede resumirse en tres áreas temáticas fundamentales: 1) la determinación de la política territorial europea, en la que se desarrolla un diagnóstico de los problemas territoriales y de intervención territorial en Europa: la definición de los objetivos y opciones políticas de la Unión Europea, básicamente equivalente al diagnóstico existente en los instrumentos de ordenación territorial; 2) el establecimiento de los «cauces» a través de los que debe materializarse la política territorial europea; y 3) las perspectivas y tendencias que debe considerar la ETE, a la hora de determinar los objetivos que ésta debe conseguir.

A pesar de señalar en su texto diversos objetivos, el fin último de la ETE se refiere a la consecución del desarrollo equilibrado y sostenible del territorio de la Comunidad Europea a través de la armonización de los tres objetivos fundamentales señalados en el propio Tratado constitutivo de la Comunidad Europea (TCCE): 1) el desarrollo competitivo y equilibrado de los territorios que la integran; 2) la conservación tanto de su medio natural como de su patrimonio cultural; y 3) la cohesión económica y social de todas sus regiones. 
Estos objetivos se coordinan en la ETE a través de la propuesta de tres directrices de desarrollo territorial que deberán ser aplicadas en cada región de la Comunidad en función de su situación específica. Tales directrices son: 1) desarrollo de un sistema urbano policéntrico y más equilibrado junto con el refuerzo de la colaboración entre los espacios urbanos y rurales; 2) fomento de las estrategias integradas de transporte y comunicación que sirvan de ayuda para el desarrollo policéntrico del territorio comunitario; y 3) desarrollo y protección de la naturaleza y del patrimonio cultural mediante una gestión inteligente que promueva la conservación y perfeccionamiento de la identidad regional, así como el mantenimiento de la diversidad natural y cultural de las regiones y ciudades de la UE.

\section{b) Naturaleza jurídica: documento soft de ordenación del territorio}

La misión de la Comunidad, señalada en el art. 2 TCE, se completa con una serie de fines, objetivos y materias señalados también en el Tratado a lo largo de su articulado, cuyo alcance definitivo, por su claro carácter dinámico, como corresponde al proceso en el que se enmarcan, queda condicionado por su subsunción en uno de los Títulos del texto normativo relativo a las políticas comunitarias que le den respaldo competencial, en función del principio de atribución de competencias al que está sometida toda la actividad de la Comunidad.

El desarrollo de esos fines, inicialmente sectoriales o funcionales, de la Comunidad Europea formalizados a través de las diferentes políticas comunitarias sectoriales y la necesidad de optimizar los efectos que sobre el territorio causan éstas - y por tanto, de su propia eficacia-, han evidenciado la necesidad de su coordinación entorno a un único instrumento: el proporcionado por la ETE. Es, por ello, que la gran aportación de la ETE se refiere a la consideración espacial de la promoción del objetivo comunitario de cohesión económica y social a través de un marco adecuado de orientación para el desarrollo de las políticas sectoriales comunitarias y en coordinación con las de los propios Estados miembros.

Sin embargo, la puesta en marcha de los objetivos de la ETE encuentran un obstáculo principal en la falta de competencia comunitaria (o europea) en materia de ordenación territorial. Es más, el instrumento mediante el cual se formaliza la ETE es un acto de los denominados «atípicos». Por ello, y a pesar del valor jurídico de esta iniciativa, parece evidente la limitación respecto de las medidas disponibles para la realización de los objetivos que en la misma se proponen.

Por tanto, y en resumen, el desarrollo territorial sostenible del territorio de la Comunidad Europea sólo es posible, al menos hasta ahora, mediante la articulación de un sistema de actuación abierta y coordinada a escala euro- 
LOS NUEVOS INSTRUMENTOS JURÍDICOS PARA EL DESARROLLO SOSTENIBLE...

pea de las políticas sectoriales territoriales de la Comunidad, y ésta sólo es posible, debido a esa falta de competencia comunitaria en materia de ordenación del territorio, a través de instrumentos jurídicos de naturaleza flexible o soft.

Precisamente, del análisis del documento de la ETE se puede claramente concluir, como ya se ha señalado, que pertenece al heterogéneo tipo de los actos atípicos de las Comunidades Europeas en tanto que no pertenece a ninguno de los enumerados como típicos por el TCE. Además, tal y como se indica en su texto, se trata de un documento jurídicamente no vinculante (no imperativo) que se constituye como marco político para mejorar la cooperación entre las distintas políticas comunitarias que tienen efectos territoriales importantes, así como entre los Estados miembros, sus regiones y ciudades. Propone «un marco adecuado de orientación para las políticas sectoriales con repercusiones territoriales de la Comunidad y de los Estados miembros, así como para las autoridades regionales y locales, a fin de conseguir un desarrollo equilibrado y sostenible del territorio europeo».

Ahora bien, como ya se ha indicado, en realidad este dato tan sólo aclara que tanto su régimen como sus efectos no se regulan por ninguno de los regímenes formalizados de los actos típicos. Por lo tanto, de lo dicho hasta ahora puede deducirse que si bien la imperatividad de la ETE, al menos en el sentido tradicional que puede darse a este concepto, está muy debilitada, esto no parece comprometer su eficacia, sobre todo en la medida en que los objetivos de política territorial queden incorporados en las demás políticas atribuidas a la Comunidad en los Tratados, como así está ocurriendo.

Pero esa eficacia no se da únicamente en el nivel europeo: la ETE está llamada a jugar, también en el nivel estatal, esa función de referibilidad, en la medida en que constituyen líneas-fuerza y criterios de ordenación sobre los que se presenta un amplio consenso y en cuya determinación han participado, también, representantes de cada uno de los Estados miembros.

Por lo tanto, resulta evidente que la ETE no se refiere a una política comunitaria articulada sobre técnicas tradicionales de intervención; lo impide, como se viene afirmando, la falta de competencia atribuida sobre esta materia en favor de la Comunidad por el Tratado. Ahora bien, en modo alguno puede reducirse este documento a un papel puramente teórico. Tiene una clara vocación práctica $\mathrm{y}$, de hecho, se encuentra en la actualidad en pleno desarrollo.

\subsection{El futuro de la planificación europea del territorio: opciones para una ordenación a escala europea a la luz del Derecho Originario actual y en ciernes}

El panorama normativo europeo actualmente vigente no ofrece posibilidad a la UE, como se ha visto, de incluir su actividad de planificación del 
territorio en el acervo comunitario. Y el futuro próximo no parece muy diferente a pesar de la terminología utilizada en el Tratado por el que se establece una Constitución para Europa (TCCE) Pero eso no significa, sin embargo, que no pueda plantearse un modelo de organización de las técnicas existentes que, en la práctica, permita a la Comunidad Europea implementar los objetivos de desarrollo sostenible sobre el territorio de la Unión, y que, por tanto, equivalga, en gran medida, al desarrollo de una Estrategia de Desarrollo Territorial Europea.

Del análisis del vigente TCE y del próximo, cuando sea ratificado definitivamente por todos los Estados miembros, se pueden construir, en principio, dos modelos válidos para la planificación del territorio a escala europea. De una parte, el que se proyecta hacia el futuro, opción A, y de otra, el que busca las técnicas a partir de lo ya vigente. Así:

\section{b.1) Opción A: ordenación del territorio formal a través de la cohe- sión territorial}

De la lectura del art. I-3.3 TCCE $^{23}$ se puede inducir que las orientaciones para el desarrollo sostenible del territorio de la UE establecidas en la ETE han evolucionado hacia la cohesión territorial:

Artículo I-3 Objetivos de la Unión

3. (...) La Unión fomentará la cohesión económica, social y territorial y la solidaridad entre los Estados miembros

Y además, la cohesión territorial se incluye, por vez primera, entre las competencias compartidas (art. I-14.2 TECE) de la UE con los Estados miembros.

El concepto de cohesión territorial rebasa la noción de cohesión económica y social, ya que su objetivo es contribuir a lograr un desarrollo más equilibrado, construir comunidades sostenibles en las zonas urbanas y rurales y procurar una mayor coherencia con otras políticas sectoriales que tienen una repercusión espacial. Este concepto implica también mejorar la integración territorial y promover la cooperación entre las regiones y dentro de ellas. Además, mejorar la cohesión territorial es una cuestión tanto de método, estableciendo la oportunidad del establecimiento de un planteamiento multidisciplinar o integrado; como de reconocimiento de los problemas particulares que presentan las diferentes circunstancias geográficas. Así, el éxito de la cohesión territorial dependerá, de una estrategia global que defina

${ }^{23}$ Para ver el texto completo del Tratado, vid. http://europa.eu.int/constitution/index_es.htm (may 2005). 
LOS NUEVOS INSTRUMENTOS JURÍDICOS PARA EL DESARROLLO SOSTENIBLE...

el marco dentro del cual se han de realizar objetivos y las acciones específicos ${ }^{24}$.

La inclusión de este nuevo concepto por el legislador comunitario podría responder a esa necesidad de la Comunidad Europea de buscar apoyos jurídicos que justifiquen su actividad planificadora. Sin embargo, el término no viene a añadir nada nuevo a la cuestión competencial ya analizada, pues en realidad tan sólo supone un refuerzo del ya existente término de cohesión económica y social, aludiendo a sus ya conocidos efectos territoriales. Por tanto, la reforma del artículo que establece los objetivos de la Comunidad Europea viene a confirmar la necesidad de avanzar en el desarrollo territorial sostenible de las regiones de Europea a través de los instrumentos soft law, que son los que, por ahora, pueden amparar jurídicamente la actividad planificadora de la EU.

En definitiva, la eficacia real y actual de los instrumentos soft law hace más difícil aún que el futuro de la planificación territorial en Europa no esté, por ahora, dirigido al desarrollo de una política de ordenación del territorio y que la evolución del proceso esté protagonizada necesariamente por esos productos informales que comienzan a aparecer, apartando a los instrumentos tradicionales que resultan demasiado rígidos para corregir el desajuste producido por los deseos de subsumir las acciones desarrolladas por la UE en la normativa comunitaria y la realidad, que se presenta siempre mucho más compleja (FARINÓS \& PAREJO, p.10).

\section{b.2) Opción B: ordenación del territorio material a través de los instrumentos soft y del MCA}

La complejidad del sistema, sin embargo, no acaba ahí, pues a la peculiar naturaleza de la actividad misma de planificación territorial desarrollada por la Comunidad se añade el ya tradicional problema asociado a la falta de transparencia en la toma de decisiones por parte de las instituciones (el denominado, en la jerga comunitaria, déficit democrático).

La UE es una peculiar y compleja organización internacional que ha evolucionado desde un sistema horizontal de cooperación interestatal hacia uno vertical multi-nivel, sin llegar a convertirse en una organización federal (HÖRE$\mathrm{TH}, 2001)$. En esta particular organización en la que los gobiernos de ámbito local, regional, multi-regional y nacional coexisten con un gobierno comunitario, el problema de legitimación en todos los niveles es obvio. Por eso, resulta imprescindible la coordinación de las relaciones verticales (multi-niveles) y horizontales (multi-sectoriales). Y esta coordinación es precisamente la

\footnotetext{
${ }^{24}$ Comunicación de la Comisión. Política de cohesión en apoyo del crecimiento y el empleo: directrices estratégicas comunitarias, 2007-2013. COM (2005) 299 final, 5 de julio de 2005. http:// www.cor.europa.eu/document/activities/lisbon/comm299-2005_part1_ext_es.pdf
} 
que se pretende lograr a través de la puesta en marcha de las buenas prácticas del Libro Blanco de la Gobernanza Europea, cuyo objetivo es la instauración de una gobernanza ${ }^{25}$ más democrática en todos los niveles de gobierno ${ }^{26}$.

Cinco son los principios que constituyen la base de una buena gobernanza y de los cambios propuestos en el Libro Blanco: apertura, participación, responsabilidad, eficacia y coherencia. Cada uno de estos principios resulta esencial para la instauración de una gobernanza más democrática en el debate sobre futuro de Europa. El concepto de gobernanza, con las características que le son inherentes, implica la necesidad de adoptar nuevos mecanismos que garanticen la pluralidad, la flexibilidad y la interacción respetando, a la vez, los principios propios del concepto (MorATA, 2005). En este sentido, los principios de la buena gobernanza promueven el uso de los de subsidiariedad y proporcionalidad, así como la calidad, efectividad y simplicidad de los procesos de decisión.

Una de las vías para la promoción de buena gobernanza pasa por el desarrollo de los documentos soft. La gobernanza demanda la utilización de mecanismos más ágiles y flexibles. Así, en éste ámbito, la promoción de los mecanismos de soft law resulta evidente. Pero la gobernanza implica también el surgimiento de nuevas formas de relación entre la pluralidad de actores necesarios para la adopción y la aplicación de las decisiones comunitarias, pues se expresa a través de una red compleja de actores, estructuras, regulaciones, competencias y responsabilidades que provocan, una vez más, la falta de transparencia en la toma de decisiones.(MORATA, 2005)

Uno de los medios más novedosos para fomentar la cooperación, el intercambio de buenas prácticas y para lograr el acuerdo de objetivos y orientaciones comunes entre los Estados miembros es el denominado «Método de Coordinación Abierta» (MCA) u «Open Method of Coordination» (OMC). Este método se emplea caso a caso y se basa en una evaluación sistemática de los progresos realizados en la materialización de los objetivos planteados, permitiendo a los Estados miembros establecer una comparación entre sus respectivos esfuerzos y aprender de la experiencia ajena. Se trata, por tanto, tal y como se definió en el Consejo Europeo de Lisboa de marzo de $2000^{27}$, de un nuevo método que resulta idóneo para alcanzar una mayor convergencia en torno a los principales objetivos de la UE.

\footnotetext{
${ }^{25}$ El concepto de Gobernanza se definió por primera vez en la Conferencia Euricities de Viena que tuvo lugar en abril del 2002 como la habilidad para organizar esfuerzos conjuntos para lograr objetivos públicos incluyendo a todos los actores implicados y a los propios ciudadanos. El propio libro de la Gobernanza define el termino como: «entendida como la organización de la acción colectiva, es la capacidad de las sociedades para dotarse de sistemas de representación, de instituciones, de procesos y de cuerpos sociales, como instrumento de control democrático, de participación en las decisiones y de responsabilidad colectiva».

${ }^{26}$ COM (2001) 428 final en http://europa.eu/eur-lex/es/com/cnc/2001/com2001_0428es01.pdf

${ }^{27}$ http://www.ehu.es/observatoriosp/pdf/lisboa.pdf
} 
LOS NUEVOS INSTRUMENTOS JURÍDICOS PARA EL DESARROLLO SOSTENIBLE..

El método consta de cuatro elementos:

1. Elaboración de directrices para la Unión, con objetivos a corto, medio y largo plazo fijados por los Estados miembros;

2. Creación de indicadores cuantitativos y cualitativos a medida que se vayan necesitando, y evaluaciones comparativas con los mejores del mundo, según las necesidades de cada Estado miembro;

3. Transferencia de las directrices europeas a las políticas regionales y nacionales, tomando en cuenta la diversidad europea. Tales reformas tendrían que integrarse en los planes de acción nacionales, y

4. Seguimiento periódico, evaluación y revisión inter-pares, organizados como procesos de aprendizaje mutuo.

El MCA se aplica en áreas que son competencia exclusiva de los Estados miembros pero en las que se han acordado algunos objetivos comunes con la UE (como la ordenación del territorio). En aquellas materias en las que la UE no tiene competencias legislativas, la consecución de tales objetivos depende únicamente de los acuerdos que se logren en los parlamentos y administraciones nacionales, quedando condicionados, por tanto, por los matices aportados por los contextos nacionales.

El MCA se ha establecido, por tanto, como la herramienta clave para la Comisión para controlar e impulsar el progreso hacia la consecución de los objetivos políticos de la UE: compromete a los Estados miembros a trabajar juntos para lograr los objetivos comunes, que son también los de la UE, sin pretender con ello la homogeneización de los ordenamientos nacionales, al tiempo que les obliga a compartir información, a establecer criterios de comparación entre unos y otros sobre la puesta en marcha de las políticas nacionales, $\mathrm{y}$, por tanto, a promocionar el aprendizaje a través de la experiencia y la búsqueda de soluciones a los problemas planteados de manera conjunta ${ }^{28}$.

El MCA permite lograr, así, la planificación del territorio en términos de sostenibilidad, conectando los objetivos de cohesión, respeto al medio ambiente y desarrollo económico, referidos en el TCE y en las directrices establecidas en la ETE.

\section{CONCLUSION: EL MODELO DE ORDENACIÓN DEL TERRITORIO A PARTIR DE UNA OPCIÓN DE NATURALEZA ECLÉCTICA}

El desarrollo territorial coherente de las regiones al amparo del TCE, hoy por hoy, sólo será posible, en principio, y debido a la falta de competencia

\footnotetext{
${ }^{28} \mathrm{http}: / /$ eucenter.wisc.edu/Conferences/OMC_Oct04/. (may 2005).
} 
de la UE en materia de ordenación del territorio, ajustando las acciones de la Comunidad al modelo B antes descrito, y articulado de la siguiente manera: en primer lugar, en lo que a la forma de trabajo, la organización en términos de competencias de los diferentes niveles territoriales implicados, etc, se refiere, a través del MCA; y en segundo lugar, en relación con la instrumentación jurídica de los acuerdos tomados por los distintos niveles a través del MCA, mediante instrumentos de naturaleza soft.

Sin embargo, el modelo así planteado queda cojo, pues no define ningún objetivo ni enumera ningún principio al que deba ajustarse la acción de la Comunidad. El método comunitario no impide, y por eso se ha incluido así en el TCCE, la definición de objetivos y acciones específicas para la cohesión territorial. Por tanto, no hay necesidad de renunciar a la actividad de cohesión propuesta en el TCCE, aunque entendida ésta, no en términos de política formal, sino como un conjunto de principios (los enunciados en el TCCE y resumidos en el término de cohesión territorial) que pueden ser formulados expresamente, pero con la garantía de su coordinación e implantación a través de mecanismos flexibles, que permitan ajustar el modelo al acervo comunitario y que son, precisamente, los propuestos en la opción B.

Este modelo, en definitiva, propone una opción ecléctica en la que las dos opciones anteriores se complementen.

Una vez más, el modelo sigue quedando abierto, en coherencia con el proceso de construcción europeo en el que éste se inserta (y quizá éste sea su mayor valor) y, así, el futuro del territorio dependerá, en gran medida, del impulso que estos instrumentos reciban del complejo sistema institucional y organizacional, llegando hasta donde indique, en cada momento, el método comunitario.

\section{BIBLIOGRAFÍA}

1. Ahonen, P., Soft Governance, Agile Union? Analysis of the Extensions of Open Coordination in 2000. European Institute of Public Administration. Maastrich, 2001. http:// unpan1.un.org/intradoc/groups/public/documents/NISPAcee/UNPAN007710.pdf.

2. DG Regional Policy. Interim Territorial Cohesion Report (preliminary results of ESPON and EU Commission Studies). Office for Official Publications of the European Communities, 2004. http://europa.eu.int/comm/regional_policy/sources/docoffic/official/reports/coheter/coheter_en.pdf.

3. European Commission. A Sustainable Europe for a BetterWorld: A European Union Strategy for Sustainable Development. COM(2001)264 final; Brussels, 15-5-2001.http:/ /europa.eu.int/eur-lex/lex/LexUriServ/site/en/com/2001/ com2001_0264en01 .pdf

4. European Commission. European Governance: A white paper. $\operatorname{COM}(2001) 428$ final, Brussels, 25-7-2001. http://europa.eu.int/eur-lex/lex/LexUriServ/ site/en/com/ 2001/ com2001_0428en01.pdf 
LOS NUEVOS INSTRUMENTOS JURÍDICOS PARA EL DESARROLLO SOSTENIBLE...

5. European Commission. Towards a Thematic Strategy for Soil Protection. COM(2002) 179 final, Brussels, 16-4-2002. http://europa.eu.int/eurlex/lex/ LexUriServ/site/en/com/ 2002/ com2002_0179en01.pdf

6. Faludi, A. The Third Cohesion Report and the European Spatial Development Perspective. European Journal of Spatial Development. http://www.nordregio.se/EJSD/-ISSN 1650-9544. March, 2004. http://www.nordregio.se/EJSD/faludioncohesion.pdf.

7. Farinós Dasí, J. Gobernanza Territorial de ámbito metropolitano. Procesos Metropolitanos en la sociedad actual. Claves y retos. http://www.upo.es/ general/centros_depart/ departamentos/geo_hist_filos/procesos_metropolitanos/documentos/ GobTerritMetropolitana.pdf (mayo 2006).

8. FARINós, J. \& PAREJO, T. Building politics through policies: Guiding trends (Legitimately?) from EU level. Paper for the ECPR Workshop on «European Spatial Politics or Spatial Policy for Europe?». 13-18 April, 2004. Uppsala, Sweeden.

9. Hildebrand And Scheid, A. Política territorial y desarrollo regional en España y Europa: Una visión comparad en vísperas del siglo XXI. Desarrollo Local y desarrollo regional frente al siglo XXI. Ciudad y Territorio. Estudios Territoriales. Vol. XXXI. Tercera Época. No 122, invierno 1999. Pags.785-807

10. H. Diamond, J. Burnham And H. TAYloR. Your heart your planet. Eartheart, Cape Town, 1991.

11. HöRETH, M. The European Commission's White Paper on governance: A «tool-kit» for closing the legitimacy gap of EU policy making?. Zentrum für Europäische Integrationsforschung Center for European Integration Studies Rheinische Friedrich WilhelmsUniversität Bonn. C 94 2001. http://www.zei.de/download/zei_dp/dp_c94_hoereth.pdf

12. KrÄMER, L. «Derecho Ambiental y Tratado de la Comunidad Europea». Marcial Pons. Barcelona, 1999. Pag.55.

13. Lázaro Araujo, L. El desarrollo local y los Fondos Estructurales. Ciudad y Territorio. Estudios Territoriales, Vol. XXXI, Tercera época, $\mathrm{n}^{\circ}$ 122, 1999. Pag. 735 a 755.

14. Montán Arella, L., 2002, The EU thematic strategy on soil protection, COM (2002) 179 final report, 275-284.

15. Morata, F. «El proyecto de constitución europea: el reto de la democracia postestatal». Revista Instituciones y Desarrollo $\mathrm{n}^{\circ} 16$ (2004) pags.276-306. http:// www.iigov.org/id/attachment.drt?art $=22655$

16. Morata, F. «Gobernanza multinivel en la Unión europea». Ed. Tirant Lo Blanch, 2005.

17. Parejo Alfonso, L. La política regional en la Comunidad Económica Europea.; incluido en García de Enterría, E.; González Campos, J. D., y Muñoz Machado, S. «Tratado de Derecho Comunitario Europeo». Volumen III. Cívitas, $1^{\circ}$ edición. Madrid, 1986. Págs. 573 y ss

18. Parejo Navajas, T. «La Estrategia Territorial Europea: la percepción comunitaria del uso del territorio». Marcial Pons, Madrid, 2004.

19. Plaza Gutiérrez, J. I. El territorio europeo ante el nuevo siglo. Revista de Estudios Europeos, $n^{\circ} 34$, mayo-agosto 2003, pags. 3-16.

20. RADAELLI, C.M. The Open Method of Coordination: a new governance architecture for the European Union?. Sieps, 2003: 1. http://www.sieps.se/_pdf/Publikationer CR20031.pdf

21. Romero González, J. y Farinós, Dasí, J. «Ordenación del Territorio y Desarrollo Territorial». Ed. Trea. Nº7.Gijón. 
22. Tulmets, E. The Introduction of the Open Method of Coordination in the European Enlargement Policy: Analysing the Impact of the New PHARE/Twinning Instrument. European Political Economy Review, Vol. 3, No. 1 (Spring 2005), pp. 54-90. http:// www.lse.ac.uk/collections/EPER/vol3/no1/tulmets.pdf.

23. Wishlade, F. and YuILl, D. Measuring disparities for area designation purposes:issues for the European Union. Regional and Industrial Policy Research Paper. Published by European Policies Research Centre. University of Strathclyde. Number 24. June 1997. http://www.eprc.strath.ac.uk/eprc/PDF_files/R24MeasDispforAreaDesigPurposes.pdf. 


\section{Jurisprudencia}

REALA-2006, núm. 302. TERESA PAREJO NAVAJAS. LOS NUEVOS INSTRUMENTOS JURÍDICOS PARA EL DESARROLLO .. 